V THE OUTSKIRTS OF GALAXIES

"My life is made miserably difficult by these facts which seem to be. getting better and better."

A. Toomre in Discussion V.2 


\title{
GALAXY HALOES AND THE MISSING MASS PROBLEM
}

Sidney van den Bergh

David Dunlap Observatory, University of Toronto

\author{
I am afraid my subject is \\ rather an exciting one and as \\ I don't like excitement, I \\ shall approach it in a gentle, \\ timid, roundabout way. \\ - Max Beerbohm
}

\section{ABSTRACT}

Available evidence on the mass-to-light ratios in binary galaxies, small clusters and in rich clusters is reviewed. The interpretation of the binary data remains ambiguous. The relative velocity of $M 81$ and $M 82$ implies that $m / L_{V}>12$. The Local Group data also appear to favour the existence of some hidden mass associated with M31 and the Galaxy. In the Virgo cluster a significant fraction of the virial mass may be concentrated in the halo of M87. In Coma the missing mass was probably stripped from the haloes of the two dominant $E$ galaxies and is now distributed throughout the cluster.

It is suggested that metal-poor stars in the galactic disc have a higher CNO abundance than do halo stars of similar metallicity. This may explain why globular cluster stars lie on taller red giant branches than do moderately high-velocity stars near the Sun. The dominant old red giant population in the disc of M31 appears to be similar to that in the Galaxy. Most of the red giants in M33, NGC 6822 and in the LMC lie on taller red giant branches than do those in the Galaxy and M31. This suggests that old evolved stars in these relatively low-luminosity galaxies have a lower heavy element abundance than do their counterparts in the galactic disc. The red giants in $M 32$ appear to be intermediate between those in M31 and M33. This supports the idea that M32 was once much more luminous than it is at present. 
1. STEllaR POPULATIONS IN THE HALO AND DISC

$1.101 \mathrm{~d}$ red giants in the Galaxy

According to ideas that were first introduced by Bade (1944) a spiral galaxy consists of a disc of (metal-rich) Population I stars that is embedded within a halo consisting of (meta1-poor) Population II stars. The most striking difference between these two populations is that halo population I stars have tall red giant branches with $-3<\mathrm{M}_{\mathrm{V}}<-2$ whereas the old giants of Population I are much fainter and have $-1<\mathrm{M}_{\mathrm{V}}<0$.

The first indication that something might be wrong with this beautifully simple picture was obtained by Keenan and Keller (1953) who showed (cf. Figure 1) that high-velocity stars in the solar vicinity have a colour-magnitude diagram resembling that of old Population I. This result was totally unexpected because high-velocity stars in the disc were supposed to be representative members of the galactic halo population.

The puzzling observations of Keenan and Keller were ignored for the next two decades. Baade believed (Sandage 1977) that the spectroscopic luminosity estimates by Keenan and Keller must have been falsified by metallicity effects.

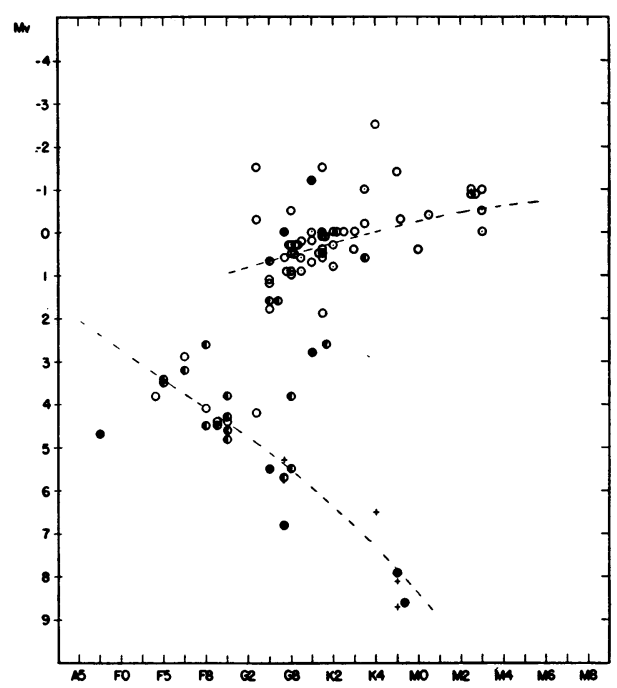

Fig. 1. Hertzsprung-Russell diagram for high-velocity $\left(\mathrm{V}>85 \mathrm{~km} \mathrm{~s}^{-1}\right)$ stars according to Keenan and Keller (1953). The figure shows that most nearby high-velocity giants lie well below the giant branch of a typical halo globular cluster. 
Striking confirmation of the results obtained by Keenan and Keller was, however, obtained by Hartwick and Hesser (1972). These authors showed (see Figure 2) that high-velocity field stars with ultraviolet excesses (which measures [Fe/H]) $\delta(U-B) \simeq+0.11$ and globular cluster giants in 47 Tucanae, for which $\langle\delta(U-B)\rangle=+0.10$, have differing red giant luminosities. This conclusion is strengthened and confirmed by the work of Demarque and McClure (1977) who show (cf. Figure 3 ) that the old open cluster NGC 2420 , for which $\delta(U-B)=$ +0.11 , has a fainter red giant branch than does the relatively metal-rich globular cluster 47 Tuc. Calculations by these authors show that the observed differences between the giants in 47 Tuc and NGC 2420 might be explained if either

(1) 47 Tuc is richer in helium than NGC 2420 by $\triangle Y \sim 0.1$ or (2) if 47 Tuc has a ten times lower Z(CNO) than does NGC 2420. Substantial support for the latter alternative is provided by the work of Hearnshaw (1972) who concludes that "irondeficient old disc stars are generally no more than marginally carbon-deficient, a result which is probably not valid for halo stars." Additional evidence for this conclusion is

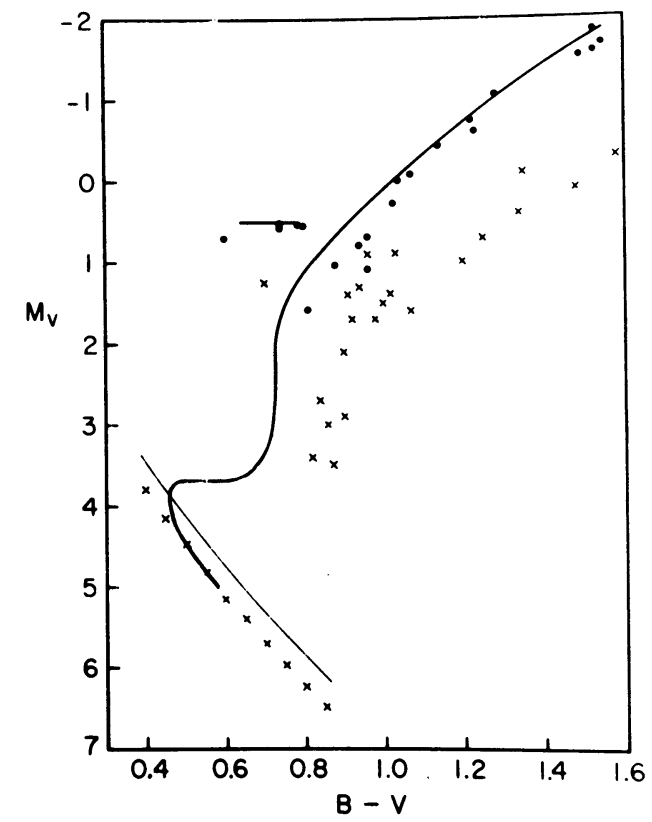

Fig. 2. Colour-magnitude diagram (Hartwick and Hesser 1972) for nearby stars (X) with $\delta(U-B) \simeq+0.11$ and for stars (•) in the globular cluster 47 Tucanae which have $\delta(U-B)=+0.10$. The figure shows that metalpoor disc stars are fainter than halo stars of similar metallicity. 


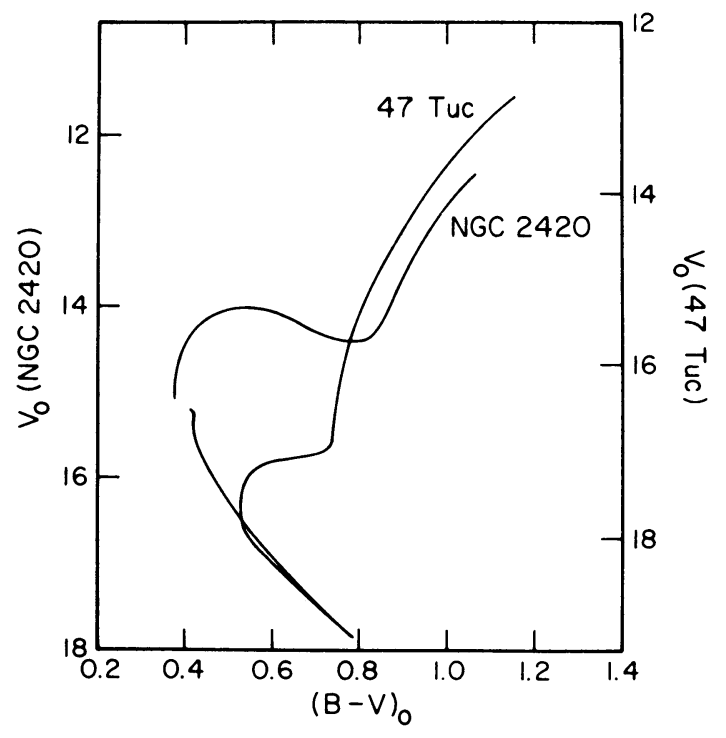

Fig. 3. Comparison of the colour-magnitude diagrams of the metal-poor open cluster NGC 2420 and the globular 47 Tucanae. The fact that 47 Tuc has the tallest. giant branch implies (cf. Demarque and Geisler 1963) that $Z(47$ Tuc) $<Z(N G C 2420)$ despite the fact that both clusters have similar $\mathrm{Fe} / \mathrm{H}$ values.

provided by the work of Hesser, Hartwick and McClure (1976) who find that the average CN strength in the giant stars of the relatively metal-rich globular cluster 47 Tucanae is lower than that in field high-velocity stars of similar metallicity.

*A possible difficulty with this point of view is that Peimbert (1973) finds that CNO/Fe is unusually high in the planetary nebula K 648 which is situated in the metal-poor globular cluster M15. The metal abundance in this cluster is down by a factor of $\sim 100$ whereas the $O$ and $\mathrm{Ne}$ abundances in $K 648$ are only $\sim 10$ times lower than they are in the Sun. An even more extreme difficulty is posed by the recentlydiscovered planetary nebula in the Fornax dwarf galaxy. Danziger et al. (1977) find the $O$ and Ar abundances in this planetary to be only two or three times lower than they are in the Orion Nebula! This result is particularly surprising because the globular clusters associated with the Fornax system (van den Bergh 1969, Danziger 1973) are exceedingly metal-poor. In view of these results it might be necessary to reconsider Peimbert's (1973) conclusion that the material in the envelopes of planetary nebulae cannot have been enriched in heavy elements by their central stars. 
Additional evidence in favour of the view that halo stars have a low CNO/Fe abundance ratio is provided by chemical abundance analyses of halo stars. One of the best known examples of a nearby star which kinematically belongs to the galactic halo is the sub-dwarf Groombridge 1830. In this unevolved star carbon and nitrogen are found to be underabundant relative to iron (Tomkin and Bell 1973). Analysis of the ${ }^{12} \mathrm{CH}$ and ${ }^{13} \mathrm{CH}$ absorption features in $\mathrm{Gmb} 1830$ by Lambert and Sneden (1977) shows that ${ }^{12} \mathrm{C} /{ }^{13} \mathrm{C} \geqslant 90$ which is similar to the ${ }^{12} \mathrm{C} /{ }^{13} \mathrm{C}$ ratio in the sun. Since nucleosynthesis in very massive objects (Wagoner, Fowler and Hoyle 1967 ) is believed to produce roughly equal amounts of ${ }^{12} \mathrm{C}$ and ${ }^{13} \mathrm{C}$ this suggests that very massive objects did not dominate the heavy element enrichment during the halo phase of galactic evolution. It therefore seems improbable that the remnants of such massive objects could contribute significantly to the "missing" halo mass.

\subsection{Red giants in other Local Group galaxies}

The bright red giants that Baade (1944) discovered in the disc of M31 are embedded in a much richer population of faint giants which contribute most of the light. A similar situation prevails in the galactic disc near the Sun and in the galactic nuclear bulge (Arp 1959, van den Bergh 1971a). Baade. (1963) refers to the bright Population II giants in the disc of the Andromeda Nebula as "the frosting on the cake".

\section{Recently Sandage (1977) has emphasised the fact that} the red giants in the disc of M33, in NGC 6822 and in IC 1613 (Sandage 1971) are all bright ( $\sim 21.5)$ and easily resolvable. Sandage points out that the surface density of these bright red stars in M33 is 2100 times greater than it is in the galactic disc near the sun. It follows that these bright red giants in $M 33$ constitute the dominant old population in the Triangulum Nebula; not a "frosting on the cake" as they do in M31 and the Galaxy.

Probably the most straightforward interpretation of this result is that the old red giants in M33, NGC 147, NGC 185, NGC 205 and NGC 6822 iie on tall red giant branches because they have low $Z$ values. The alternative hypothesis that these stars lie on tall branches because they have high Y values appears to be ruled out by the observation that the present helium abundance in M33 and in NGC 6822 (Peimbert and Spinrad $1970 \mathrm{a}, \mathrm{b})$ is slightly below that prevailing in galactic HII regions.

In IC 1613 and in the Small Magellanic Cloud the interpretation of the observations is rendered uncertain by the 
possibility that a major fraction of the bright red giants might resemble those in the intermediate-age SMC cluster NGC 419 .

A comparison between recent abundance determinations for HII regions in the SMC, the LMC and the Galaxy is given in Table I. These data suggest that $Z(G a 1 a x y) / Z(L M C) \simeq 3$ and $\mathrm{Z}(\mathrm{Galaxy}) / \mathrm{Z}(\mathrm{SMC}) \simeq 10$. These results are consistent with the weakening of the lines of $\mathrm{N}, \mathrm{O}, \mathrm{Si}$ (and probably of $\mathrm{Mg}$ and $\mathrm{C}$ as we11) that has been reported by Osmer (1973) in the spectra of LMC and SMC stars. Little direct evidence is available on the $\mathrm{Fe}$ abundance of stars in the Magellanic Clouds. From DDO photometry of the giant stars in the intermediate-age LMC cluster NGC 2209 Gascoigne et al. (1976) obtain [Fe/H] $\sim 1.0$. From synthetic spectra Bell and Parsons (1972) find that stars with Teff $\sim 6000^{\circ} \mathrm{K}$ will become $\sim 0.1$ mag bluer in B-V if their metal abundance is reduced by a factor of 4 . From a compilation of data on all Cepheids with photoelectric B-V observations (van den Bergh 1977a) it appears that [Fe/H] $\gtrsim-0.6$ for the LMC and $[\mathrm{Fe} / \mathrm{H}] \leqslant-0.6$ for the SMC. These results indicate that both the abundances of the CNO group and of the metals are presently substantially lower in the Magellanic Clouds than they are in solar-type stars. This suggests that the old stars in the Magellanic Clouds must also have significantly lower $Z$ values than do similar stars in the Galaxy. This conclusion is supported by Hardy's (1977) observations of stars in the disc of the LMC. These data, which are plotted in Figure 4, show that most of the old disc giants in the LMC are situated on evolutionary tracks that lie between the giant branches of the SMC globular NGC 121 and the relatively metal-rich galactic globular 47 Tuc. Hardy's data suggest, but do not yet prove conclusively, that the disc of the LMC contains few if any of the high $Z$ M67 and NGC 188 type of red giants with $\mathrm{M}_{\mathrm{V}} \sim 0$.

\section{TABLE I}

HEAVY ELEMENT ABUNDANCES *

\begin{tabular}{lllll}
\hline \hline Region & He & N & 0 & Ne \\
\hline SMC HII & 10.89 & 6.4 & 8.0 & 7.3 \\
LMC HII & 10.92 & 7.0 & 8.5 & 7.8 \\
Orion Nebula & 11.00 & 7.7 & 8.89 & 8.15 \\
\hline
\end{tabular}

*Entries in the table are $\log [\mathrm{N}(\mathrm{X}) / \mathrm{N}(\mathrm{H})]+12.00$ taken from a compilation by Dufour and Killen (1977). 


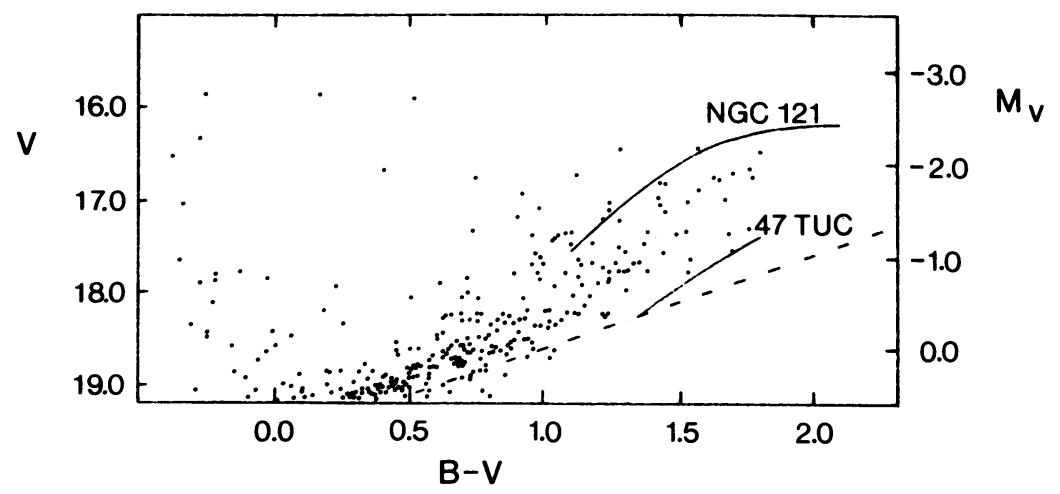

Fig. 4. Colour-magnitude diagram for a region in the disc of the LMC (Hardy 1977). The figure shows that most giants lie on giant branches that are intermediate between those of NGC 121 and 47 Tucanae. The dashed line shows the limit of completeness of the data.

The results obtained above may be summarised as follows: The galaxies of the Local Group may be divided into two groups according to the properties of their dominant red giant populations. In M33, the LMC, NGC 6822, NGC 147, NGC 185, NGC 205 and probably in the SMC and IC 1613 as well the red giants are bright indicating a relatively low heavy element abundance. In the Galaxy and M3 I the dominant red giant population consists of old faint giants that are composed of material with a relatively high $Z$ value. The red giants in M32 seem to have characteristics that are intermediate between those of the high-luminosity and lowluminosity members of the Local Group. This result is consistent with the work by Faber (1973a, b) who finds that M32 has lost a considerable amount of mass by tidal stripping resulting from encounters with M31. Its CN + Mg index suggests that M32 may now be $\sim 5$ times 1 ess 1 uminous than it was originally.

In the dwarf galaxies of the Local Group and in the halo of the Galaxy low metallicity and low CNO (and hence low Z) go together. This contrasts with the situation in the galactic disc in which $Z$ (CNO) appears to have remained constant while $Z$ (Fe) continued to rise (cf. Figure 5). These results show that the traditional practice of using the terms metallicity $(\mathrm{Fe} / \mathrm{H})$. and heavy element abundance interchangeably is both misleading and incorrect. 


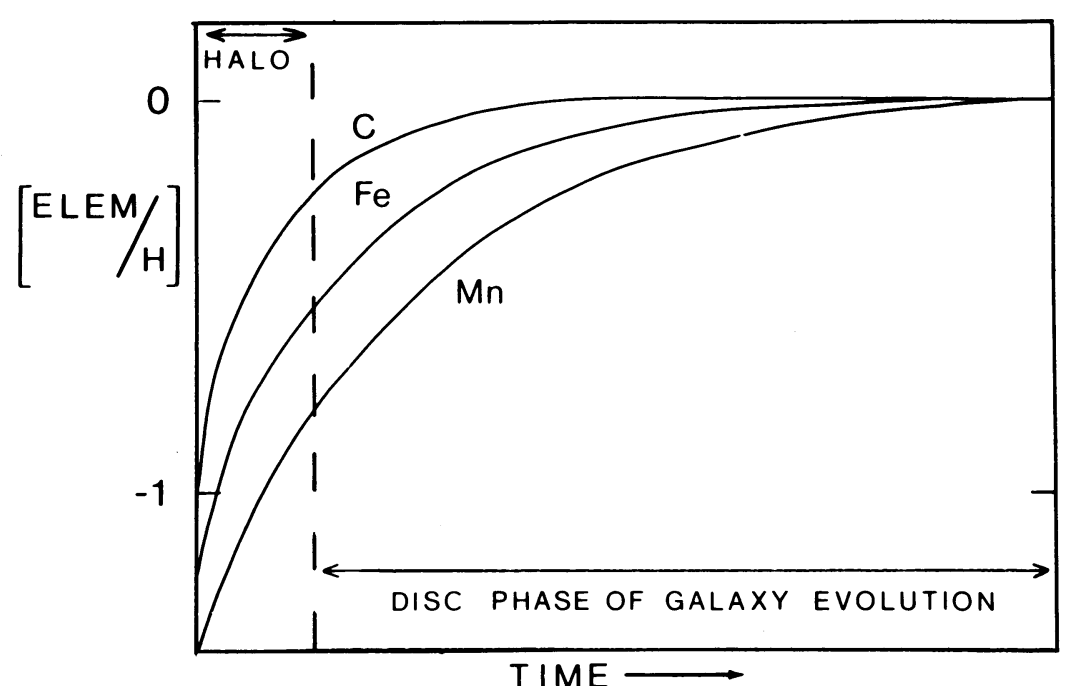

Fig. 5. Schematic plot illustrating the time-dependent changes of the $\mathrm{C}, \mathrm{Fe}$ and $\mathrm{Mn}$ abundances. in the galactic disc according to Hearnshaw (1972). The diagram shows that moderately metal-poor disc stars might have almost normal carbon abundances.

\section{EVIDENCE FOR MISSING MASS}

\subsection{Missing mass near the Sun}

In a recent review Gliese (1976) shows that the total density of known stars and interstellar material near the Sun amounts to $0.07 \leqslant \rho \leqslant 0.09 m_{\odot} \mathrm{pc}^{-3}$. This value appears to be significantly smalier than the dynamically-determined local mass density of $0.15 \mathrm{~m}_{0} \mathrm{pc}^{-3}$ (Oort 1965). It is profoundly disturbing to find that as much as one third to one half of the mass in the nearest region of space may exist in some unknown form. According to Schmidt (1975) the local density of halo stars, defined as objects with tangential velocities larger than $250 \mathrm{~km} \mathrm{~s}^{-1}$, is $1.7 \times 10^{-4} \mathrm{~m}_{\mathrm{\rho}} \mathrm{pc}^{-3}$. This represents only $\sim 1 \times 10^{-3}$ of the total local density. It follows that the missing mass near the sun cannot be in the form of normal $\left(0.1<m / m_{\odot}<1\right)$ halo stars.

Theoretical work by Kalnajs (1972) and by Ostriker and Peebles (1973) appears to show that "cold disc" galaxies are unstable unless they are embedded in massive haloes. To date direct optical evidence for the existence of such massive haloes is still lacking. Available colour measurements show that the haloes of most galaxies are bluer than are their 
main bodies (cf. Simkin 1975, Strom and Strom 1977). The most straightforward interpretation of this observation is that globular cluster-like stars do and that faint M-type dwarfs do not contribute significantly to the integrated light of galaxy haloes.

\subsection{Velocity dispersion of galactic globular clusters}

Hartwick and Sargent (1977) have obtained new radial velocities for distant palomar-type globulars and for dwarf spheroidal companions to the Galaxy. For eleven such objects, which are situated at an effective distance of $\sim 75$ kpc, Hartwick and Sargent get a total galactic mass of $(9 \pm 2) \times 10^{11} m_{\odot}$ if the cluster velocities are assumed isotropic and $(3.2 \pm 0.6) \times 10^{11} m_{\odot}$ if the cluster orbits are purely radial. These values appear to be significantly larger than those obtained from conventional galactic models (eg. Schmidt 1956, Innanen 1973) which yield masses in the range $1-2 \times 10^{11} m_{\odot}$. This conclusion is, however, quite uncertain because the number of outlying objects for which radial velocities are available is quite small. Radial velocities of outlying clusters in M31 may soon provide a value for the mass of the halo of the Andromeda Nebula.

\subsection{Rotation curves of spirals}

$21-\mathrm{cm}$ observations of neutral hydrogen gas have made it possible to extend the rotation curves of galaxies to much greater radial distances than was possible previously. Such observations (Roberts 1975, Krumm and Salpeter 1977) appear to show that a substantial number of spirals have rotation curves that are essentially flat out to the largest distances to which neutral hydrogen can be detected. Additional observations are, however, needed to prove that the observed rotation curves are not significantly affected by non-circular motions or by "spillover". Such spillover might be important in some cases where an antenna points toward the faint outer regions of a galaxy while receiving emission from the bright central area of that galaxy into a side lobe.

\subsection{Binary galaxies}

In principle observations of the velocity differences of pairs of galaxies (Page 1952) should be able to provide information on the total masses of galaxies. Due to complex selection effects and differing methods of analysis different authors still obtain quite widely divergent results.

Assuming $\mathrm{H}=50 \mathrm{~km} \mathrm{~s} \mathrm{Mpc}^{-1}$ Karachentsev (1977) obtains $\mathrm{m} / \mathrm{L} \sim 7$ for spiral-spiral pairs whereas Turner (1977) gets $\mathrm{m} / \mathrm{L} \sim 65$. The former result is quite compatible with that obtained from rotation curves whereas the latter value is 
consistent with massive halo models. It is unlikely that the difference between these results is due to a difference in the mean separations of the pairs studied by these two authors. For Karachentsev's spiral-spiral pairs the mean separation of the components is $50 \mathrm{kpc}$, compared to a mean separation of $64 \mathrm{kpc}$ for Turner's field galaxy sample. In the case of elliptical galaxies (which are usually located in clusters), the determination of $\mathrm{m} / \mathrm{L}$ ratios is particularly tricky because it is often difficult to distinguish between optical and physical pairs.

\subsection{Small clusters of galaxies}

The density contrast between small clusters and the general background field of galaxies is low. As a result small cluster samples are always in danger of contamination by field galaxies. Simple-minded attempts to reduce such contamination by using galaxy velocities as a selection criterion are self-defeating since they result in truncation of the Maxwellian velocity distribution. This leads to an artificial reduction in the velocity dispersion and hence to m/L ratios that are too low. Such a bias may, at least in part, account for the low mass-to-light ratios obtained by Materne and Tammann (1974). An additional complication is that the observed velocity dispersion in some density enhancements on the sky can be reduced (Tully and Fisher 1976) by assuming that they consist of two or more clusters at different distances that happen to be superimposed on each other. Finally a number of small clusters have "crossing times" that are longer than the Hubble time (Jackson 1975). Such groups need not be gravitationally bound.

\section{From a recent analysis of data on 39 groups of galaxies} Gott and Turner (1977) obtain $m / L \sim 140$. For the richest subset in their sample these authors obtain $\mathrm{m} / \mathrm{L} \approx 200$ whereas they find that $M / L \sim 65$ is more appropriate for small groups. High mass-to-1ight ratios for many (but not all) small groups are also derived by Rood et al. (1970) and by Rood and Dickel (1976). Einasto et al. (1975) have argued that these high $m / L$ ratios are due to hidden mass that is located in the haloes ("coronas") of the giant galaxies that dominate some of these groups. Additional information on the $\mathrm{m} / \mathrm{L}$ ratios in small clusters may be obtained from observations of the Local Group and the M81 group.

Perhaps the most remarkable feature of the Local Group (Kahn and Woltjer 1959) is that M3l and the Galaxy (which probably contain most of the mass) are presently approaching each other. If $M 31$ and the Galaxy were formed close together $1.3 \times 10^{10}$ yr ago, reached a maximum separation of $850 \mathrm{kpc}$ $8.5 \times 10^{9}$ yr ago and are now falling towards each other at 
$70 \mathrm{~km} \mathrm{~s}^{-1}$ then their combined mass must be $\sim 2.9 \times 10^{12} \mathrm{~m}_{0}$ from which $M / L \sim 125$ (Gunn 1974). The conclusion that the dynamics of the Local Group is dominated by missing mass can on ly be avoided (Herbst 1975) if the galactic rotational velocity of the Sun is $\gtrsim 290 \mathrm{~km} \mathrm{~s}^{-1}$, which appears to be unacceptably high. This problem is, however, slightly alleviated by the fact that all of the companions to the Andromeda Nebula (see Table II) for which radial velocities are available are redshifted relative to M3l itself.

On the basis of the frequency with which supergiant galaxies occur in nearby space van den Bergh (1971b) finds that the a priori probability that M3l and the Galaxy are unrelated galaxies that are passing each other like ships in the night is only $\sim 4$ percent. Furthermore, such a situation is physically implausible (Silk and Lea 1973) because decay of primaeval random motions in an expanding Universe would be expected to reduce the relative velocities of clusters to at most a few $\mathrm{km} \mathrm{s}^{-1}$ at the present epoch.

The M81 group is dominated by M81 and M82. M81 has a radial velocity $\mathrm{V}=-44 \mathrm{~km} \mathrm{~s}^{-1}\left(\mathrm{~V}_{0}=+95 \mathrm{~km} \mathrm{~s}^{-1}\right)$ (de Vaucouleurs, de Vaucouleurs and Corwin 1976). For M82 neutral hydrogen observations yield V $=+205 \mathrm{~km} \mathrm{~s}^{-1}$ (Crutcher, Rogstad and Chu 1977) and optical observations (O'Connell and Mangano 1977) give $+200.6 \pm 2.4 \mathrm{~km} \mathrm{~s}^{-1}$. Adopting $\mathrm{V}=+203 \mathrm{~km} \mathrm{~s}^{-1}\left(\mathrm{~V}_{\mathrm{O}}=+34 \underline{\mathrm{km} \mathrm{s}^{-1}}\right)$ for M82 yields a velocity difference $\Delta V_{0}=250 \mathrm{~km} \mathrm{~s}^{-1}$. For a closed orbit

$$
\Delta \mathrm{V}_{\mathrm{O}}{ }^{2} \mathrm{P}<2 \mathrm{G}\left(\mathrm{M}_{81}+\mathrm{M}_{82}\right) \text {. }
$$

At a distance of $3.25 \mathrm{Mpc}$ (Tammann and Sandage 1968) the projected separation $P$ of $M 81$ and $M 82$ is $36 \mathrm{kpc}$ so that $\mathrm{M}_{81}+\mathrm{M}_{82}>2.6 \times 10^{11} \mathrm{~m}_{\odot}$. Since $\mathrm{L}_{81}+\mathrm{L}_{82}=2.2 \times 10^{10} \mathrm{~L}_{\odot}$ (Holmberg 1958) it follows that $\mathrm{M}_{\mathrm{S}} \mathrm{L}_{\mathrm{V}}>12$.

TABLE I I

RADIAL VELOCITIES OF COMPANIONS TO M31

\begin{tabular}{cccc}
\hline Ga1axy & $V\left(\mathrm{~km} \mathrm{~s}^{-1}\right)$ & $\mathrm{V}_{\mathrm{O}}\left(\mathrm{km} \mathrm{s}^{-1}\right)$ & $\Delta \mathrm{V}_{\mathrm{O}}\left(\mathrm{km} \mathrm{s}^{-1}\right)$ \\
\hline M31 & -299 & -61 & 0 \\
M33 & -180 & +5 & +66 \\
NGC 221 & -195 & +43 & +104 \\
NGC 205 & -239 & +1 & +62 \\
NGC 185 & -208 & +41 & +102 \\
NGC 147 & -157 & +95 & +156 \\
\hline
\end{tabular}




\subsection{Rich clusters of galaxies}

Application of the Virial theorem to rich clusters of galaxies appears to give overwhelming support to the notion that the dynamics of such clusters is dominated by hidden mass. Recent determinations of the $7 n / L$ ratios in the nearest giant clusters (van den Bergh 1977b) are summarised in $\mathrm{Tab} 1 \mathrm{e}$ III.

$X$-ray observations of the Virgo cluster (Gorenstein et al. 1976) show that the diffuse emission from this cluster, is centred on the giant elliptical M87 to an accuracy of $\sim z$. The $x$-ray emission comes from a region with a radius of $\sim 1$, which is significantly smaller than the $\sim 6$ radius of the Virgo cluster core. If the observed $x$-rays are generated by hot gas that is confined by the potential well of M87 then the total mass of this galaxy must be a significant fraction of the Virial mass of the Virgo cluster (Bahcall and Sarazin 1977, Mathews 1977). This observation constitutes one of the strongest pieces of evidence in favour of the view that some galaxies may have very massive haloes (Ostriker, Peebles and Yahil 1974). The situation in Virgo appears to differ from that prevailing in the Coma cluster in which the low degree of concentration of massive galaxies towards the cluster centre (Rood 1965, White 1977) shows that most of the cluster mass is not bound to individual galaxies. For the intergalactic material in the Coma cluster Melnik, White and Hoessel (1977) find $\mathrm{m} / \mathrm{L} \gtrsim 1000$. Possibly this material was originally located in the haloes of the two dominant galaxies in the Coma cluster and was subsequently stripped off by tidal interactions (cf. Gallagher and Ostriker 1972). Richstone (1976) has shown that as much as ninety percent of the total mass of galaxies in a typical rich cluster might be stripped off by tidal shocks during one Hubble time.

\section{TAB LE I I I}

MASS-TO-LIGHT RATIOS IN THE NEAREST RICH CLUSTERS

\begin{tabular}{lr}
\hline Cluster & $\mathrm{m} / \mathrm{L}_{\mathrm{V}}{ }^{*}$ \\
\hline & \\
Perseus & 350 \\
Coma & 200 \\
Virgo & 250 \\
\hline${ }_{\mathrm{H}}=50 \mathrm{~km} \mathrm{~s}{ }^{-1} \mathrm{Mpc}^{-1}$ assumed.
\end{tabular}


The importance of tidal interactions on the outer structure of E galaxies is beautifully illustrated by Kormendy's (1977) photometry. His results show that elliptical galaxies with companions have much more extended haloes than do isolated ellipticals. Additional support for the view that the outer structure of $E$ galaxies is affected by tidal interactions is provided by recent CTIO 4-m observations (van den Bergh 1978). This work shows that the envelopes of "cD" galaxies in poor clusters (Morgan, Kayser and. White 1975) almost all fall into two classes: (A) objects with faint symmetrical envelopes that were presumably produced during tidal encounters that took place long ago and (B) galaxies embedded in bright asymmetrical envelopes that were probably formed by recent tidal encounters.

Finally deep photographs of radio sources associated with nearby elliptical galaxies such as Centaurus A (NGC 5128 $=$ Figure 6) and Fornax A (NGC $1316=$ Figure 7) show that these galaxies are embedded in extended haloes that exhibit a rather chaotic structure. Neither the physical nature of these haloes nor their origins are presently understood.

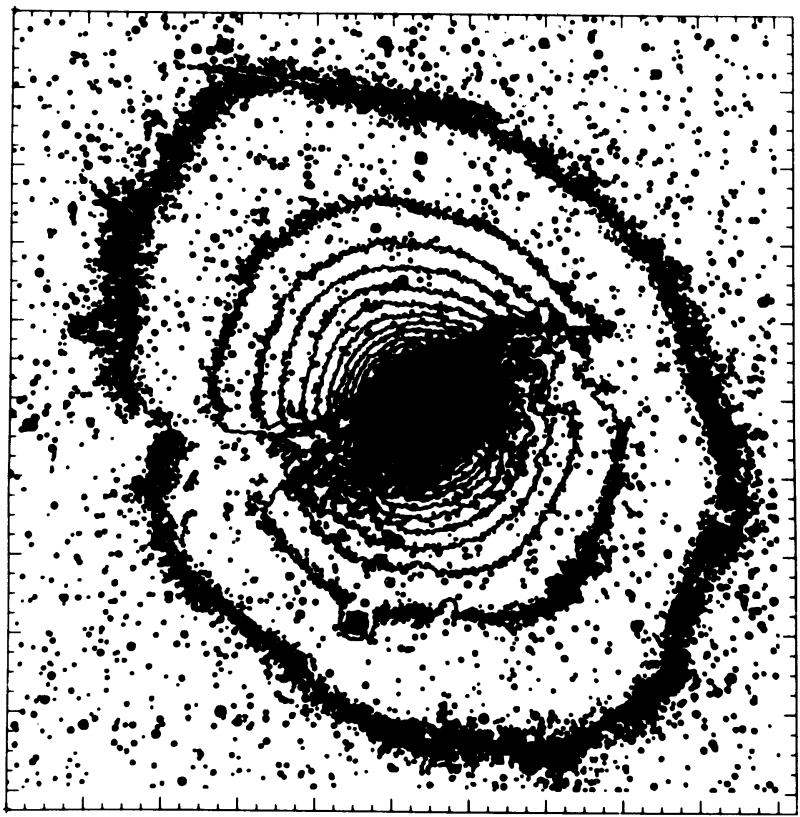

Fig. 6. Outer isophotes of NGC 5128 in yel1ow (103aD+ GG495) light according to Dufour and van den Bergh (1978). 


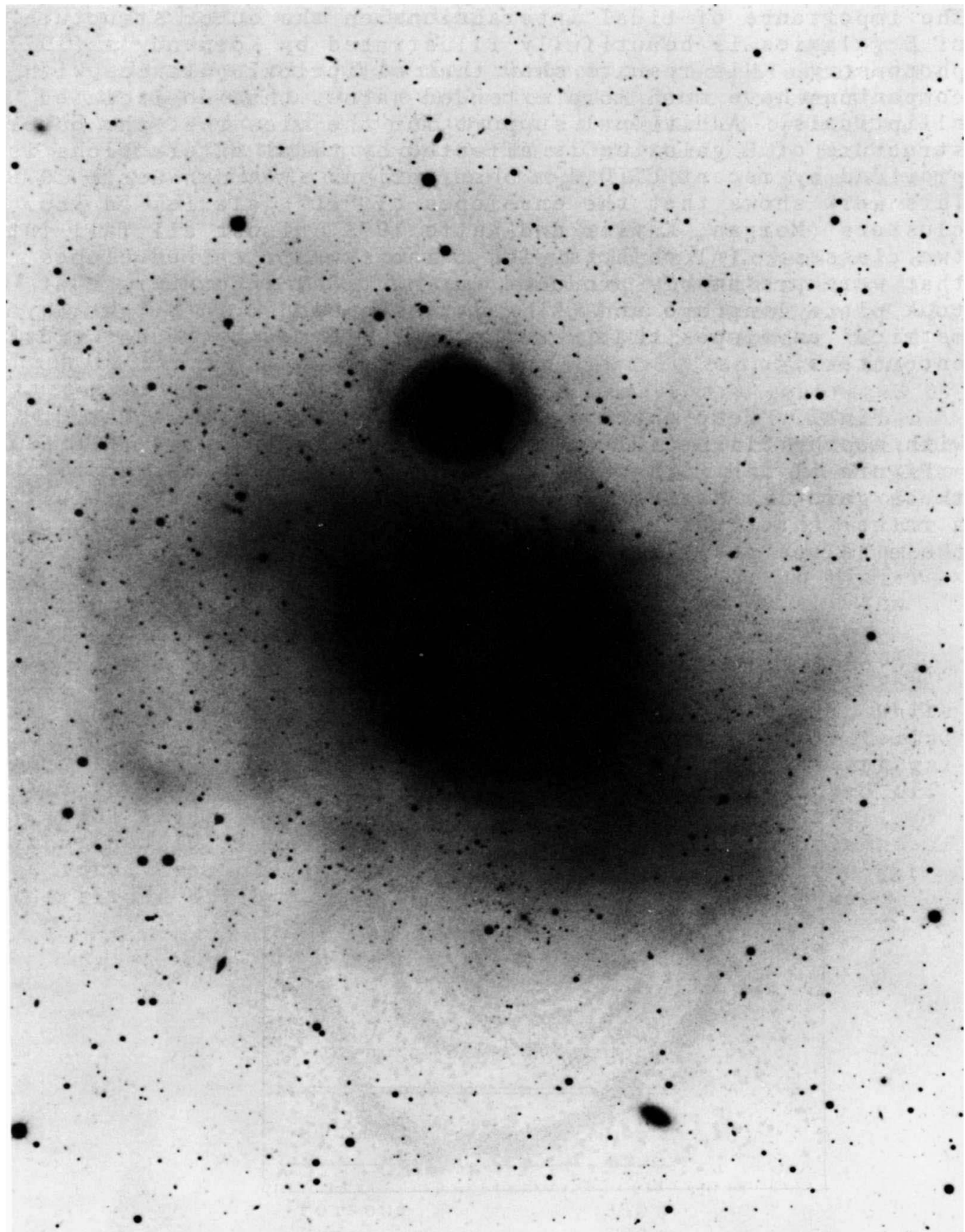

Fig. 7. Photograph of the halo of NGC 1316 (Fornax A) obtained in red 1ight (127-04+GG495) with the CTIO 4-m reflector. 


\section{REFERENCES}

Arp, H. C.: 1959, Astron. J. 64, 33 .

Baade, W.: 1944, Astrophys. J. 100, 137 .

Baade, W.: 1963, Evolution of Stars and Galaxies, Cambridge, Harvard University Press.

Bahcall, J. N. and Sarazin, C. L.: 1977, Astrophys. J. Letters 213 , L99.

Be11, R. A. and Parsons, S. B.: 1972 , Astrophys. Letters 12 , 5 .

Crutcher, R. M., Rogstad, D. H., and Chu, K.: 1977

(preprint).

Danziger, I. J.: 1973, Astrophys. J. 181, 641 .

Danziger, I. J., Dopita, M. A., Hawarden, T. G., and Webster, B. L.: 1977 , Astrophys. J. (in press).

Demarque, P. and Geisler, J. E.: 1963, Astrophys. J. 137 , 1102 .

Demarque, P. and McClure, R. D.: 1977, Astrophys. J. 213 , 716 .

de Vaucouleurs, G., de Vaucouleurs, A., and Corwin, H. G.:

1976 , second Reference Catalogue of Bright Galaxies,

Austin, University of Texas Press.

Dufour, R. J. and Killen, R. M.: 1977, Astrophys. J. 211 , 68.

Dufour, R. J. and van den Bergh, S.: 1978 (in preparation) .

Einasto, J., Kaasik, A., Kalamees, P., and Vennik, J.: 1975, Astron. Astrophys. 40, 161 .

Faber, S. M.: $1973 \mathrm{a}$, Astrophys. J. $179,423$.

Faber, S. M.: 1973b, Astrophys. J. 179, 731 .

Gallagher, J. S. and Ostriker, J. P.: 1972, Astron. J. 77, 288 .

Gascoigne, S. C. B., Norris, J., Bessel1, M. S., Hyland, A., R., and Visvanathan, N.: 1976 (preprint).

Gliese, W.: 1976, in E. K. Kharadze (ed.), Proceedings of the Third European Astronomical Meeting of the I.A.U., Abastumani, Georgian Academy of Sciences, p. 463.

Gorenstein, P., Topka, K., Fabricant, D., and Harnden, F. R.: $1976, B u 11$. Am. Astron. SoC. 8, 553 .

Gott, J. R. and Turner, E. L.: 1977, Astrophys. J. 213, 309 .

Gunn, J. E.: 1974, Comments on Astrophys. Space Sci. 6, 7 . Hardy, E.: 1977, Astrophys. J. 211, 718 .

Hartwick, F. D. A. and Hesser, J. E.: 1972, Publ. Astron. Soc. Pacific 84,813 .

Hartwick, F. D. A. and Sargent, W. L. W. : 1977 (preprint). Hearnshaw, J. B.: 1972, Mem. Roy. Astron. Soc. $77,55$.

Herbst, W.: 1975, Publ. Astron. Soc. Pacific 87,827 .

Hesser, J. E., Hartwick, F. D. A., and McClure, R. D.: 1976 , Astrophys. J. Letters 207 , L113.

Holmberg, E.: 1958, Lund Medd. Ser. 2, no. 136.

Innanen, K. A.: 1973, Astrophys. Space Sci. 22, 39. 
Jackson, J. C.: 1975 , Monthly Notices Roy. Astron. Soc. 173, $4 \mathrm{lp}$.

Kahn, F. D. and Woltjer, L.: 1959, Astrophys. J. 130, 705 .

Kalnajs, A. J.: 1972, Astrophys. J. 175, 63 .

Karachentsev, I. D. : 1977, in C. Balkowski and B. E.

Westerlund (eds.), Décalages Vers le Rouge et Expansion de I'Univers, Paris, C.N.R.S., p. 321 .

Keenan, P. C. and Keller, G.: 1953, Astrophys. J. 117, 241.

Kormendy, J.: 1977, Astrophys. J. (in press).

Krumm, N. and Salpeter, E. E.: 1977, Astron. Astrophys. 56, 465 .

Lambert, D. L. and Sneden, C.: 1977, Astrophys. J . $215,597$.

Materne, J. and Tammann, G. A.: 1974, Astron. Astrophys. 37 , 383 .

Mathews, W. G.: 1977, Lick Obs. Bull. no. 766.

Melnik, J., White, S. D. M., and Hoessel, J.: 1977, Monthly Notices Roy. Astron. Soc. $180,207$.

Morgan, W. W., Kayser, S., and White, R. A.: 1975, Astrophys. J. 199,545 .

0'Conne11, R. W. and Mangano, J. J.: 1977 (preprint).

Oort, J. H.: 1965, in A. Blaauw and M. Schmidt (eds.), Galactic structure, Chicago, University of Chicago Press, p. 455 .

Osmer, P. S.: 1973, Astrophys. J. Letters 184, L127.

Ostriker, J. P. and Peebles, P. J. E.: 1973, Astrophys. J. 186,467 .

Ostriker, J. P., Peebles, P. J. E., and Yahil, A. : 1974 , Astrophys. J. Letters 193 , L1.

Page, T.: 1952, Astrophys. J. 116, 63 .

Peimbert, M.: 1973 , Mem. Soc. Roy. des Sciences de Liège $6^{\text {th }}$ ser. 5, 307 .

Peimbert, M. and Spinrad, H.: 1970a, Astrophys. J. 159, 809 .

Peimbert, M. and Spinrad, H.: 1970b, Astron. Astrophys. 7, 311 .

Richstone, D. 0.: 1976, Astrophys. J. 204, 642 .

Roberts, M. S.: 1975 , in A. Hayli (ed.), Dynamics of stellar systems, Dordrecht, Reide1 Pub1. Co., p. 331 .

Rood, H. J.: 1965, University of Michigan (unpublished Ph.D. Thesis).

Rood, H. J. and Dickel, J. R. : 1976, Astrophys. J. 205, 346 .

Rood, H. J., Rothman, V. C. A., and Turnrose, B. E.: 1970 , Astrophys. J. 162, 411 .

Sandage, A. R.: 1971, Astrophys. J. 166, 13 .

Sandage, A. R.: 1977 , paper presented at the Pomona Meetings of the Astronomical Society of the Pacific, June 21 , 1977 .

Schmidt, M.: 1956, Bull. Astron. Inst. Neth. 13, 15.

Schmidt, M.: 1975, Astrophys. J. 202, 22.

Silk, J. and Lea, S.: 1973, Astrophys. J. $180,669$.

Simkin, S. M.: 1975, Dudley Obs. Repr. no. 9, p. 401. 
Strom, K. M. and Strom, S. E.: 1977, in B. Tinsley (ed.), The Evolution of Galaxies and Stellar Populations (in press).

Tammann, G. A. and Sandage, A. R.: 1968, Astrophys. J. 151, 825 .

Tomkin, J. and Bell, R. A.: 1973, Monthly Notices Roy. Astron. Soc. 163,117 .

Tul1y, R. B. and Fisher, J. R.: 1976, in E. K. Kharadze (ed.), Proceedings of the Third European Astronomical Meeting of the I.A.U., Abastumani, Georgian Academy of Sciences, p. 481 .

Turner, E. L.: 1977, in C. Balkowski and B. E. Westerlund (eds.), Décalages Vers le Rouge et Expansion de I'Univers, Paris, C.N.R.S., p. 337.

van den Bergh, S.: 1969, Astrophys. J. Suppl. 19, 145 .

van den Bergh, S.: 1971a, Astron. J. 76, 1082 .

van den Bergh, S.: 1971b, Astron. Astrophys. 11, 154.

van den Bergh, S.: $1977 \mathrm{a}$, in C. Balkowski and B. E.

Westerlund (eds.), Décalages Vers le Rouge et Expansion

de I'Univers, Paris, C.N.R.S., p. 13.

van den Bergh, S.: 1977b, Vistas Astron. 21, 71.

van den Bergh, S.: 1978 (in preparation).

Wagoner, R. V., Fowler, W. A., and Hoyle, F.: 1967, Astrophys. J. 148,3 .

White, S. D. M.: 1977 , Monthly Notices Roy. Astron. Soc. 179,33 .

\section{DISCUSSION FOLLOWING REVIEW V.1 GIVEN BY S. VAN DEN BERGH}

OORT: You mentioned the possibility that the relative velocity of M31 and the Galaxy might indicate a chance encounter. However, there are other, independent galaxies in the Local Group, for instance NGC $6 \overline{822}$, which make a chance encounter unlikely.

FREEMAN: Perhaps I could add here that the whole question of what actually is in the Local Group, and what sort of velocity dispersion you derive from this mass, is a controversial one. Sandage has recently made a solar motion solution where he feels justified in throwing out all galaxies at a velocity that deviates more than $3 \sigma$ from the solar motion solution. Then, of course, the whole mass of our Local Group can shrink abruptly. This point is far from solved.

Furthermore, I want to reinforce Dr. van den Bergh's remarks about problems in deriving $\mathrm{M} / \mathrm{L}$ from binary galaxies. Turner analysed his sample by two methods: Page's which gave M/L $\sim 3$, and his own which gave $\mathrm{M} / \mathrm{L} \sim 40$. The problem here is in the procedure, primarily. A definitive paper, on how to do this properly, will appear soon.

M.S. ROBERTS: S. Peterson has recently completed a study of binary galaxies combining Turner's observations with additional new data he obtained from $21-\mathrm{cm}$ line observations. He finds $\mathrm{M} / \mathrm{L} \sim 25\left(\mathrm{H}=50 \mathrm{~km} \mathrm{~s}^{-1}\right.$ 
$\mathrm{Mpc}^{-1}$ ) for spiral pairs. This value is essentially constant with angular separation. The total mass of a pair increases with separation up to $\sim 100 \mathrm{kpc}$ and then remains constant at $\sim 2 \times 10^{12} \mathrm{M}_{\odot}$ for larger separations. Thus these data do not indicate an extensive massive halo.

TULLY: We have done a virial analysis on a dozen or so nearby small groups, making use of a large body of new $21-\mathrm{cm}$ velocities. In a large majority of cases the virial masses are comparable to masses found from rotation curves, although we concede the possibility they might be a factor of two greater. The most remarkable exception was the M81 group where the virial theorem suggests a very large mass. However, almost al1 the kinetic and potential energy are in the pair M81-M82. If these two are taken as a single entity and the virial analysis is repeated then there is no difficulty with the proposition that the group is stable with conventional masses.

REES: Recent numerical simulations by Simon White at Cambridge indicate that, if the galaxies in binaries had halos extensive enough to be almost merging, they would spiral together in little more than one orbital timescale. This suggests that - for galaxies in binaries at least - the "halos" do not extend beyond about $50 \mathrm{kpc}$.

HUCHTMEIER: SENSITIVE OBSERVATIONS OF HI ENVELOPES OF LATE-TYPE GALAXIES

Neutral hydrogen has been observed with a sensitivity of a few times $10^{18} \mathrm{~cm}^{-2}$ with the $100-\mathrm{m}$ radio telescope (Effelsberg) in the environment of the nearby late-type galaxies Holmberg I, IC 1613, M33, Holmberg II, WLM, M101, NGC 2366, NGC 3109, Sextans A, M83, IC 10, partially in NGC 2403 and 6822 . The ratios of HI-radius to Holmbergradius are $1.3,1.6,2.2,2.4,3.6,3.6,3.7,4.5,5.8,6.2$, and 9 , respectively. The diameters of the HI envelopes correspond to linear distances of $5,11,38,200$, and $230 \mathrm{kpc}$ in the case of Holmberg I, WLM, M33, $\mathrm{M1O1}$, and $\mathrm{M} 83$, respectively.

For M1O1 about 30\% of the HI mass of $24 \times 10^{9} \mathrm{M} \odot$ is found outside of the Holmberg diameter. $1.5 \times 10^{9} \mathrm{M}_{\odot}$ are associated with the southern companion NGC 5474. The velocity field in the HI envelope is in agreement with the assumption that most of the material in the envelope is in corotation with the galaxy.

There are a few indications for a possible gravitational interaction between M1O1 and its companions. Between M101 and NGC 5474 an HI link is seen in two velocity channels (separated by $16.5 \mathrm{~km} / \mathrm{s}$ ) at a surface density of $5 \times 10^{18} \mathrm{~cm}^{-2}$ (beam averaged), which is well above possible side lobe contributions. A few of the M101 velocity channels show asymmetries of the kind expected from interactions.

The area of this 1 ink and an area close to the companion dwarf ga1axy NGC 5477 show considerably broader profiles $(\Delta \mathrm{v}>60 \mathrm{~km} / \mathrm{s}$ instead of $<40 \mathrm{~km} / \mathrm{s}$ ) than nearby regions. This is probab1y the effect of different HI complexes along the line of sight and suggests bridge-1ike features between these galaxies. However, the outer contours of the HI distribution of $\mathrm{M} 101$ are considerably more symmetric than the central 
part which is strongly disturbed.

LANDECKER: Considering the known sidelobe levels of the telescope, what is the dynamic range attainable in such measurements?

HUCHTMEIER: The outermost detected HI has a column density of about $3 \times 10^{18} \mathrm{~cm}^{-2}$, and the accumulating effects of sidelobe errors for the channels where the strongest HI signals have been observed is about half that value.

GIOVANELLI: Does the velocity field of M101 behave regularly throughout the HI distribution?

HUCHTMEIER: The velocity field is very regular. With the present resolution it does not show any deviations from an extrapolated field of circular rotation. 